them in their boats and covers problems from cardiac arrest through hypothermi decompression sickness and nitrogen narcosis and other diving conditions, to heit exhaustion and jelly fish stings. There are also sections on radio communication helicopter transfer and of course telephone numbers for advice. While not being $: \frac{a}{a}$ medical manual, it is a good little book and, if it is going to be carried by divers, then \& E departments would benefit from having a copy, so that they know what it contairs and what divers are being advised.

However, there are at present two omissions from the book. Firstly, on page 4 it tel $\frac{\overline{\bar{f}}}{\mathrm{\rho}}$ divers, in the event of an accident, to bring the book with them to hospital to aid medicat personnel not familiar with diving medicine but there is no guidance in the book fơ inexperienced casualty officers when faced with a diving accident victim. A single page of information with a few essential dos and don'ts, such as not using Entonox and fillin the cuffs of endotracheal tubes and catheters with water rather than air if the patient 8 likely to be recompressed, and advice to give patients $100 \%$ oxygen until they can get a decompression chamber would be a valuable addition to the book. The last omission 8 that we tend not to see divers with decompression sickness, because they go straight $\overrightarrow{0}$ the decompression chamber, I presume, though we see plenty of divers on summer weekends with painful reddened ears or perforated eardrums and a paragraph or two

about this would also add to the value of the book.
A second edition incorporating these two extra pages would transform the book frow being useful addition to an A \& E department library to being a desirable one!

H. R. GULY

Consultant, Accident \& Emergency Dept,

Freedom Fields Hospital,

Plymouth, England

\title{
Manual of Pediatric Emergencies
}

Edited by Joseph R. ZANGA

Churchill Livingstone, Edinburgh. 1987. £32.00.

As one would expect with any wide-ranging multi-author book, this volume is a mixtu of the good, the mediocre and, occasionally, the inaccurate.

The editor offers the volume to 'Pediatricians, Emergency Department Physicians and House Staff' and expects it to be used as a refresher in quiet moments or as instruction manual when very sick children are admitted. It is for the former rather tha the latter purpose that I think it would be useful as there is no substitute for experience or previous clear instruction from the A \& E consultant when seriously ill childre present.

The book starts well with a chapter by the editor entitled 'Children are not littif adults' with some useful physiological ranges for different ages of child. I found the layout of the book a particularly good feature. Each chapter, although by a different author, has been constructed to be of a similar layout. Large amounts of material are 
presented on the whole very clearly in list form. However, the lists are presented in such a way as to be readable and easy to follow.

As the book is wholly North American there are understandable transatlantic differences. These include the usual differences in pharmacological drug names (for example, Acetaminophen for Paracetamol, Epinephrine for adrenaline and so forth) and, of course, differences in medico-legal procedures. Rather more subtle is the suggestion that, in the States, more management of any specific emergency conditions is carried out in the emergency room than would be usual in this country, although it may only be the author's thoroughness in suggesting management options that gives this impression. In addition, there are, in some conditions, clear differences in practice between the United States and this country. This is noticeable in the management of asthma where the use of inhaled Beta-agonists has not been so widely accepted in the States as it has here.

The chapter on cardiopulmonary resuscitation in the child was particularly well done. The one on toxicological emergencies contained far too little information and the chapter on seizures went too deeply into evaluation of the EEG than is necessary in the accident and emergency department.

I was surprised to see any mention of haemolytic emergencies completely omitted from the book, particularly surprising in an American book where one would expect sickle-cell disease and G6PD to be a not uncommon problem.

The chapter on child abuse and neglect (again by the editor) is clearly presented but, again, the management side of the advice given goes further than one would expect the casualty officer to be skilled in. Again, the involvement of other colleagues (here the police surgeon) would be usual and casualty officers would obviously need to bear in mind their own local policies.

In the management of meningitis, I would have liked to have seen it made clearer that a lumbar puncture can be a dangerous procedure in a child with an altered conscious state. Even with an open fontanelle coning can occur. In a very seriously ill child with obvious meningitis, treatment should be instituted and the puncture deferred until the child does not have clinical signs of raised intracranial pressure. Similarly, I would like to have seen more emphasis on the fact that the treatment of meningococcaemia is extremely urgent and nothing should delay treatment of the patient with penicillin intravenously, except the prior drawing of blood through the same needle for culture.

In conclusion, this is a good attempt at a very wide subject and the information is well presented. I would recommend a study of the diagnostic parts of this book to casualty officers but would suggest caution in carrying out the management recommendations.

BARBARA M. PHILLIP

Accident and Emergency Department, Booth Hill Children's Hospital, Manchester, England 